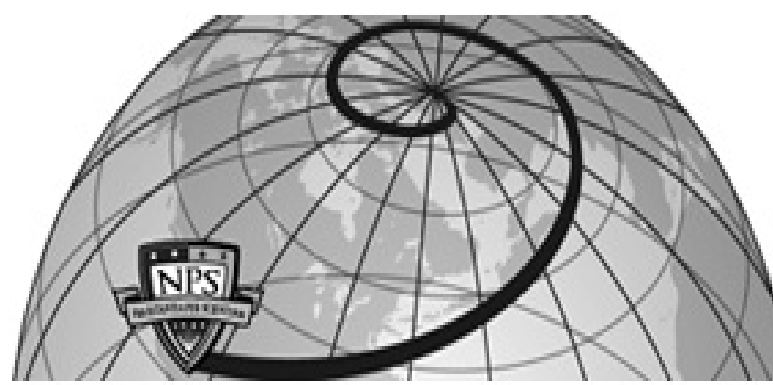

Calhoun: The NPS Institutional Archive DSpace Repository

\title{
Asynchronous Data Fusion For AUV Navigation Via Heuristic Fuzzy Filtering Techniques
}

An, P.E.; Healey, A.J.; Park, J.; Smith, S.M.

An, P. E., Healey, A. J., Park, J., Smith, S. M., "Asynchronous Data Fusion For AUV Navigation Via Heuristic Fuzzy Filtering Techniques", Proceedings IEEE, Oceans 97, Halifax, Oct. 1997 IEEE CD-ROM 0-7803-4111-2

https://hdl.handle.net/10945/36048

This publication is a work of the U.S. Government as defined in Title 17, United States Code, Section 101. Copyright protection is not available for this work in the United States.

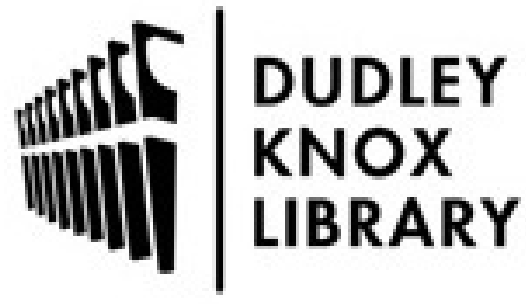

http://www.nps.edu/library
Calhoun is the Naval Postgraduate School's public access digital repository for research materials and institutional publications created by the NPS community. Calhoun is named for Professor of Mathematics Guy K. Calhoun, NPS's first appointed -- and published -- scholarly author.

Dudley Knox Library / Naval Postgraduate School 411 Dyer Road / 1 University Circle Monterey, California USA 93943 


\title{
Asynchronous Data Fusion For AUV Navigation Via Heuristic Fuzzy Filtering Techniques
}

\author{
P. E. An $\ddagger$, A. J. Healey $\dagger$, J. Park $\ddagger$, S. M. Smith $\ddagger$ \\ 抽epartment of Ocean Engineering \\ Florida Atlantic University, Boca Raton, FL 33431 \\ Email: ean@oe.fau.edu \\ $†$ Department of Mechanical Engineering \\ Naval Postgraduate School, Monterey, CA 93943
}

\begin{abstract}
This paper presents a heuristic fuzzy position estimation technique for autonomous underwater vehicle navigation. The heuristic estimator performs asynchronous data fusion of all sensor measurements based on their relative confidence levels, and then nonlinearly combines the fused information with the INS estimates via fuzzy filtering techniques. In this paper, the basis and implementation of the estimator will be described, and navigation results will be presented based on the heuristic estimator. In addition, performance comparison based on the heuristic estimator and those based on extended Kalman filters will be reported in our companion paper, and the results are expected to provide insights into the pros and cons of individual methods in terms of computational cost, steady-state and convergence characteristics for bias estimation.
\end{abstract}

\section{Introduction}

With rapid progress in COTS sensors and electronics technology, miniaturized autonomous underwater vehicles (AUV) have reached an acceptable level of maturity and reliability which can be capitalized on their use for oceanographic and military applications $[5,6]$. Examples include spatio-temporal surveys during clandestine oceanographic and mine counter-measure operations in shallow-water environments. Without requiring any tethering support, the dynamic stability and data sampling quality can be much improved. In addition, multiple small AUVs can be deployed simultaneously to traverse in different regions without necessitating one-to-one surface ships, and this results in higher data sampling efficiency [7]. To truly characterize four-dimensonal ocean dynamics autonomously, high-precision underwater navigation is a technically challenging issue because vehicle localization must be done onboard. While differential GPS sensor technology provides a good solution for surface navigation [2], underwater navigation still remains a challenging problem especially when sonar position beacons are unavailable. The main goal of this paper is to present a novel heuristic data fusion which collates different and independent asynchronous position sensors together, and nonlinearly gain schedules them with the onboard INS system. One important objective of our study is to evaluate the effectiveness of the algorithmic performance based on computationally-intensive modelbased approach $[3,8]$ versus heuristic sensor-based ap- proach with respect to steady-state and convergence performance of bias estimation. This evaluation will be reported in our companion paper. This paper is organized as follows: the next section briefly describes the sensor and capability of the Ocean Explorer AUV. Section 3 presents the heuristic navigation architecture and algorithms currently implemented on the OEX. Section 4 presents navigation results based on GPSdoppler aided INS system, and finally the last section provides concluding remarks.

\section{Ocean Explorer}

The OEX AUV is a small and untethered autonomous underwater vehicle. It is $7 \mathrm{ft}$ long (extendable to 10 $\mathrm{ft}$ ) and maximum diameter is 21 ". The hull is based on a modified Gertler's Series 58 Model 4154 with a modular mid-section for interfacing swappable payloads. The cruciform control surfaces are aft-mounted, and are replaceable with larger fins if a 3 ' mid-section is inserted. In air, the vehicle weighs approximately $4001 \mathrm{bs}$, and is designed to be neutrally buoyant. The OEX cruises at a speed of 3 knots (a range of 2-5 knots), and can execute any pre-programmed mission continuously for approximately 12 hours before Ni-Cad batteries are to be re-charged. Note that the vehicle mission can be re-programmed while the OEX is 
in water, thereby increasing the operational efficiency. In terms of navigational capability, the OEX houses 1) an acoustic doppler sensor which measures altitude and vehicle velocity with respect to either water column or ground; 2) a Watson AHRS unit which measures Euler angles, tri-axial body rates and acceleration; and 3) a differential GPS receiver unit; 4) RF ethernet and 5) CTD sensor instrument which provides depth measurement. All sensors and actuators onboard were each attached to their individual Neuron Modules and the low-level control communication is done using the LonTalk protocol. High-level planning and control processes are carried out on the VxWorks real-time operating system (with $68030 \mathrm{CPU}$ ) and the communication among processes is data-driven using a shared memory archiecture with semaphores and dedicated I/O function calls (see $[5,6,7]$ for more detail).

\section{Heuristic Navigation Archi- tecture}

Figure 1 shows a general architecture for setting up the navigational module in the OEX. In this architecture, there are arbiters created for position sensors, attitude sensors and motion sensors. This set up is desirable because many existing AUVs incorporate multiple sensors performing same functions, and it is thus beneficial to fuse all information to obtain the best navigation estimates. In cases of sensor failure, these arbiters will reconfigure in order to complete time-critical missions.

Inputs to the position sensor arbiter are absolute AUV position measurements, which can be based on (D)GPS and various forms of baseline sonar fixes. These measurements are particularly valuable because of their drift-free properties over a longer time scale, as compared to the dead-reckoning position estimate. However, over a shorter time scale, the DGPS fixes might be correlated thus introducing undesirable position error for compensation. It is thus important to carefully sample these fixes so that the signal-to-noise ratio is maximized. In addition, measurements from all sensors are generally unavailable at every time sampling instant, and a strategy is thus needed to combine these asynchronous measurements before routing the result to the heuristic position estimator.

To combine the position measurements, each sensor is assigned a confidence value which characterizes its expected variance in error about the true value. For an example, typical DGPS fixes can have $1-5 \mathrm{~m}$ range error $(\sigma)$ together with horizontal dillution of precision (HDOP) uncertainty due to satellite geometry. The total position error introduced in terms of rootmean-square value is $H D O P \times \sigma$. It should be noted that while the range error is generally hard to quantify, the HDOP profile can be readily obtained from any receiver, and thus it should be accounted for in the position estimator otherwise position error might be compromised. Once a suitable time sampling interval is chosen, the output of the position sensor arbiter is given as

$$
X=\sum Z_{i} \cdot C_{i}
$$

where $X$ is the arbiter output, $Z_{i}$ is the $i$ th sensor output, and $C_{i}$ is its confidence value which has accounted for both the range error and baseline geometry of satellites or sonar beacons. Note that a constraint of $\sum_{i=1}^{N} C_{i} \equiv 1$ is imposed on the confidence values, and thus $C_{i}$ can be loosely interpreted as prior probability that the $i$ th sensor is correct. Among these position sensors only the horizontal profiles are sought because of their much improved range error tolerances and the fact that the depth information can be obtained directly from the doppler sonar. Now the remaining task is to determine these $C_{i}$.

One important objective in this paper is to strive for a practical estimation algorithm which is not computational intensive but yet provides theoretically sound approach in performing data fusion. To have a reasonable trade-off, consider the following condition in which the unknown quantity to be estimated is a constant, $X^{*}$, and is being observed by $N$ independent sensors. It can be easily shown [1] that, in such a case, an expression of the minimum mean-squared error estimate and the resulting error variance can be established

$$
\begin{aligned}
C_{i} & =\frac{\Pi_{j=1}^{N, i \neq j} e_{j}^{2}}{P} \\
C & =E\left[\left(X-X^{*}\right)^{2}\right]=\left[\sum_{i=1}^{N} \frac{1}{e_{i}^{2}}\right]^{-1} \\
e_{i} & =\sigma_{i} h_{i}
\end{aligned}
$$

where $e_{i}$ is the expected position error standard deviation for the $i$ th sensor. $\sigma_{i}$ and $h_{i}$ represent the range error and HDOP profile for the $i$ th sensor. $\Pi$ is the product operator, and $P$ corresponds to a sum of $N$ unique combinations of variance terms where each term consists of a product of $N-1$ variances. $C$ corresponds to the overall error variance. For illustrative purposes, consider $N=3$,

$$
\begin{aligned}
C_{1} & =\frac{e_{2}^{2} e_{3}^{2}}{e_{1}^{2} e_{2}^{2}+e_{1}^{2} e_{3}^{2}+e_{2}^{2} e_{3}^{2}} \\
C_{2} & =\frac{e_{1}^{2} e_{3}^{2}}{e_{1}^{2} e_{2}^{2}+e_{1}^{2} e_{3}^{2}+e_{2}^{2} e_{3}^{2}} \\
C_{3} & =\frac{e_{1}^{2} e_{2}^{2}}{e_{1}^{2} e_{2}^{2}+e_{1}^{2} e_{3}^{2}+e_{2}^{2} e_{3}^{2}} \\
C & =\left[\frac{1}{e_{1}^{2}}+\frac{1}{e_{2}^{2}}+\frac{1}{e_{3}^{2}}\right]^{-1}
\end{aligned}
$$

It should be noted that these confidence values are only meaningful if the statistical properties of the sensor measurements are stationary, and the state dynamics are negligible although the effect of state dynamics will be studied and reported in our companion paper.

Similarly, the attitude sensor arbiter generates the best estimate of the Euler angles and body-fixed angular rates. This arbiter setting enables direct comparison in performances using different compasses without requiring any code modification. The arbiter outputs are then routed to both the heuristic position estimator and motion sensor arbiter.

In the motion sensor arbiter, the doppler-based ground and water velocities are compensated based 
on the CTD output. At present, the default sound speed was set to $1500 \mathrm{~m} / \mathrm{s}$ and correction was made in post-processing. Typical variation in sound speed with respect to the default setting can be as high as $2 \%$, and this error must be accounted for during long underwater transits.

\subsection{Heuristic Position Estimator (HPE)}

Figure 2 shows a block diagram of the heuristic estimator which considers only the ground speed as a special case in order to simplify explanation. $X_{n}$ and $X_{e}$ correspond to the absolute position measurements defined in north-east co-ordinates given a pre-defined origin. Similarly, $\hat{X}_{n}$ and $\hat{X}_{e}$ correspond to the position estimator outputs using the same origin. In the diagram, the outputs of the attitude sensor arbiter are used to transform the ground speed from the bodyfixed frame to geographical frame. If there is no position measurement, the position estimator is then reduced to a dead-reckoner, and no offsets are needed for $\hat{X}_{n}$ and $\hat{X}_{e}$. If there exists at least one position update, the position residual error (the difference between the measurement and heuristic position estimator output) is then nonlinearly gain scheduled in order to generate appropriate offsets for $\hat{X}_{n}$ and $\hat{X}_{e}$ for each of the absolute position measurement, and the nonlinear $K_{e}$ and $K_{n}$ are given as

$$
\begin{aligned}
\delta_{n} & =X_{n}-\hat{X}_{n} \\
\delta_{e} & =X_{e}-\hat{X}_{e} \\
\hat{X}_{n}^{+}(k) & =\hat{X}_{n}^{-}(k)+K_{n} \delta_{n} \\
\hat{X}_{e}^{+}(k) & =\hat{X}_{e}^{-}(k)+K_{e} \delta_{e} \\
K_{e}\left(\delta_{e}\right) & =1-\exp ^{-\delta_{e}^{2} / C^{2}} \\
K_{n}\left(\delta_{n}\right) & =1-\exp ^{-\delta_{n}^{2} / C^{2}}
\end{aligned}
$$

From these expressions, it can be easily seen that when there is small residual error with respect to $C$, the HPE discounts the contribution from the measurement as primarily sensor uncertainty, and resorts to deadreckoning which generally provides a smoother position estimate. In the case the residual error becomes relatively significant, the HPE assumes that the error is largely caused by the motion sensor drift, and thus weighs heavily on the measurement. Figure 3 provides a flow chart of the detailed operation at every navigation cycle. By examining the flow chart, one can see that when both water and ground speed are available, currents will be estimated in the geographical frame after transforming the speed difference. In cases where there is a loss of bottom track at large altitude, the current estimate can be used together with the water speed to estimate the ground speed over a relatively short time scale within which the estimated currents magnitude is slowly decaying. Two important observations can be made regarding the HPE performance. Firstly, the HPE does not explicitly estimate the sensor biases and thus the navigation performance without any position measurements is no better than that of standard dead-reckoning estimator. Secondly, the nonlinear error correcting gains are heuristically defined based on the assumption of gaussian sensor noise distribution. It should be noted that in a companion paper, the same data sets were filtered using extended 2D Kalman filters ${ }^{1}$, and the objective for such arrangement is to compare the performance between using numerically intensive algorithms with bias estimation and the heuristic algorithms without bias estimation but relying on position measurements. The critical factors to be considered for the comparison include the convergence rate and steady state error in the bias estimates and magnitude of drift induced between fixes. Among these data sets, only DGPS position fixes were available, and thus the true heading reference can only be deduced when the OEX was on surface. It should be noted that, to accomodate asynchronous data fusion, the position estimator integration time step was chosen to be the smallest interval with respect to all sensor throughputs (among the results presented, the sampling rate was set to $8 \mathrm{~Hz}$, $\sigma_{\text {dgps }}=2$ and $\sigma_{\text {gps }}=30$. In cases when there is no position fix (es) available during some time interval, no offsets will be added to the position estimator outputs.

\section{Results}

Navigation results presented in this paper were based on a combination of the Motorola GPS receiver, Acupoint differential receiver and the doppler-aided INS system. To provide important insights, the GPS performance was first characterized in a controlled environment, followed by the evaluation of the heuristic position estimation performance which was based on at-sea data.

Figure 4 and 5 show respectively the time histories of fixes and the converted X-Y excursion (at a latitude of 26 degrees, Boca Raton, Florida) collected in a single experiment during which the OEX was located in a circular salt-water pool of $10 \mathrm{ft}$ diameter, and the GPS and FM antennas were held above the surface. It should be noted that the OEX was not rigidly mounted thus slight vehicle movement should be expected although it was constrained to within $2 \mathrm{ft}$ in radius, which is well within the rated DGPS accuracy. The choice of pool test was made so as to minimize any effect associated with the controlled environment.

Time history plots consist of longitude (meter), latitude (meter) and the GPS status (0: NO GPS, 1: GPS, 2: DGPS). It can be seen that the distribution of GPS and DGPS fixes were fairly uniform, and during the time when DGPS fixes were available (around $2500 \mathrm{sec}$ ), there was minimal fluctuation in both latitude and longitude. On the contrary, when only the GPS fixes were available (around 250sec), there was significant fluctuation observed, and the apparent velocity speed was as high as 0.1 knot, as indicated by the time histories data. It is thus important to consider the effect of GPS correlated noise and sampling rate when estimating currents over a short temporal scale. Nevertheless, the navigation performance can be also critically dependent on the specific trial loca-

\footnotetext{
${ }^{1}$ It is worth mentioning that standard Kalman filters cannot be directled applied to navigation problems because of the kinematics transform between co-ordinate frames.
} 
tion, and in particular, the effect of GPS noise will become relatively insignificant when the OEX is to be operated in Florida currents of 3-5 knots, and in deep water regions with only water speed measurements.

From the XY plot, it can be seen that all errors were confined to within a 5 meter square area. To evaluate the worst case sampling interval, one can consider the following ideal condition under which the OEX cruises horizontally along for $T$ seconds at $u$ knots with $\delta u$ speed error and $\delta \psi$ heading error. The position error, $\delta X$, can be crudely approximated as

$$
\delta X=T \sqrt{u^{2} \delta \psi^{2}+\delta u^{2}}
$$

For an example, given DGPS fixes with 5 meter error, the OEX cruises at 2 knots with $1 \%$ speed error and 2 degree heading error, $T$ is approximately 3 minutes. That means, the maximum sampling interval is 3 minutes or position estimation performance will be significantly compromised. On the contrary, $T$ should not be much smaller than 3 minutes otherwise the estimator will be significantly biased with GPS noise.

Figure 6 and 7 compare the INS way-point navigation performance based on 1) doppler returns (dash line) and 2) GPS + doppler (solid line). In these figures, ' $\mathrm{X}$ ' and ' $\mathrm{O}$ ' represent a differential and regular GPS fix respectively, and in both missions the OEX was started at the origin. During these missions, the OEX was underwater most of the time, and commanded to surface during some specified cornerings in order to obtain fixes, and thus position drift due to doppler and attitude sensors can be easily observed, as compared to the DGPS fixes as the only source of reference. At the end of the last eastward leg in Figure 6 , there was a significant discrepancy between the position estimator and DGPS measurement (approximately $1.5 \%$ error based on 50 meters after 3300 meters transect). It is worth mentioning that during previous OEX surface trials with continuous DGPS fixes (not included in this paper), the doppler measurement error was found to lie within $1 \%$, and the additional error herein was believed to be caused mainly by the heading measurement with internal magnetic interference. Figure 7 presents results of a 3 -hr mission covering $15 \mathrm{~km}$ transect. Sporadic fixes can be seen in the figure which corresponds to the OEX surfacing maneuvers. Among these fixes, maximum discrepancy between the position estimator and GPS fixes was found at location [-50 east, 150 north], and also it can be seen that the position estimator responds less to these GPS fixes, but much more to the DGPS fixes obtained immediately afterwards. By observation, the discrepancy was approximately $100 \mathrm{~m}$ since the last update (6 legs of transect away $\approx 3300 \mathrm{~m}$ ), and thus the error was approximately $3 \%$. It should be noted that $100 \mathrm{~m}$ is within the limit of the GPS error deviation, and the result suggests that accurate navigation does not necessarily require frequent surfacing, as expected from previous discussion.

\section{$5 \quad$ Remarks}

In this paper, the basis and implementation of the heuristic position estimator has been described, and navigation results which were based on this estimator has been illustrated. This paper was also emphasized that the navigation performance must be evaluated with respect to the entire system as a whole, rather than an isolated entity. Thus, factors such as mission requirements, systems reconfigurability, costeffectiveness and computing resources must be altogether considered with regard to the constrainted resources currently available on the OEX. Results of such evaluation can then provide important insights into the effectiveness of sensor-based and model-based approach. For long underwater missions without any sonar positional beacons and with minimal surfacing frequency, estimation of sensor biases might be required for high precision navigation, and such information must be extracted. Extended Kalman filters were applied to those data sets with known heading bias, and the results will be reported in our companion paper in terms of steady-state error and convergence performance. Other than focusing on the position sensor measurements, parallel work on improving our existing INS system in currently underway such as localizing the main source of interference, demagnetizing the battery housing, and developing a more accurate deviation table based on a fiber-optic gyro (Andrews Corp) as short-term heading reference.

\section{References}

[1] A. Gelb, Applied Optimal Estimation, ed. A. Gelb, MIT Press, 1986.

[2] G. Lachapelle, M.E. Cannon, G. Lu, B. Loncarevic Shipborne GPS Attitude Determination During MMST-93, IEEE Journal of Oceanic Engineering, Vol.21, No.1, pp.100-105, January, 1996.

[3] R.B. McGhee, J.R. Clynch, A..J. Healey, S.H. Kwak, D.P. Brutzman. X.P. Yun, N.A. Norton, R.H. Whalen, E.R. Bachmann, D.L. Gay, W.R. Schubert An Experimental Study of an Integrated GPS/INS System for Shallow-Water AUV Navigation ( $S A N S$ ), Proceeding of Unmanned Untethered Submersible Technology Symposium, 15p, Durham, New Hampshire, September, 1995.

[4] M.J. Rendas, I.M.G. Lourtie Hybrid Navigation System for Long Range Operation, AUV 94, pp.353-359, 1994.

[5] S.M. Smith, S.E. Dunn, T.L. Hopkins, K. Heeb, T. Pantelakis The Applications of a Modular AUV to Coastal Oceanography: Case Study on the Ocean Explorer, IEEE Oceans 95 Conference, pp.1423-1432, San Diego, CA, October, 1995.

[6] S.M. Smith, K. Ganesan, T. Flanigan, L. Marquis The Intelligent Distributed Control System Architecture in the Ocean Voyager II and Ocean Explorer Vehicle, 9th International Symposium on Untethered Submersible Technology, pp. 395-405, Durham, NH, September, 1995.

[7] S.M. Smith, S.E. Dunn, P.E. An Data Collection with Multiple AUVs for Coastal Oceanography, Oceanology International 96, pp.263-279, Brighton, U.K, March, 1996. 
[8] K.P. Schwarz, M. Wei, M.V. Gelderen Aided Versus Embedded: A Comparison of Two Approaches to GPS/INS Integration, pp. 314-322, 1994.

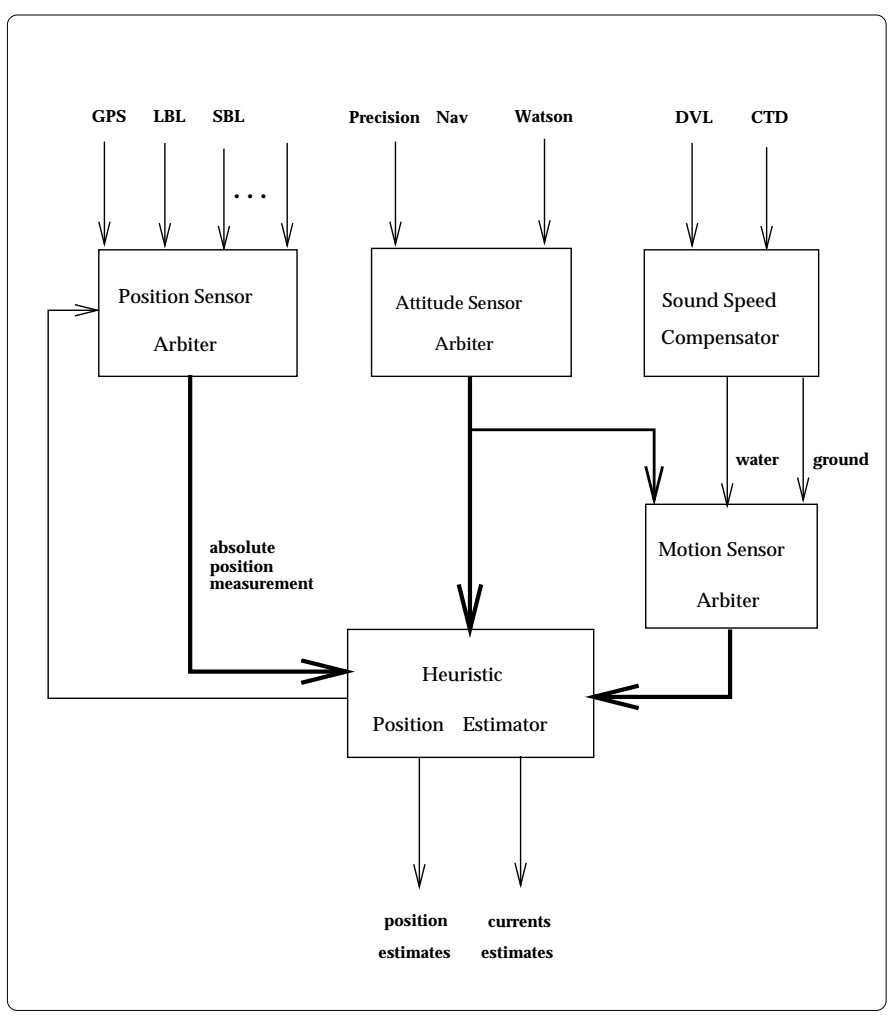

Figure 1: High-level arbiters for setting up heuristic position estimator.

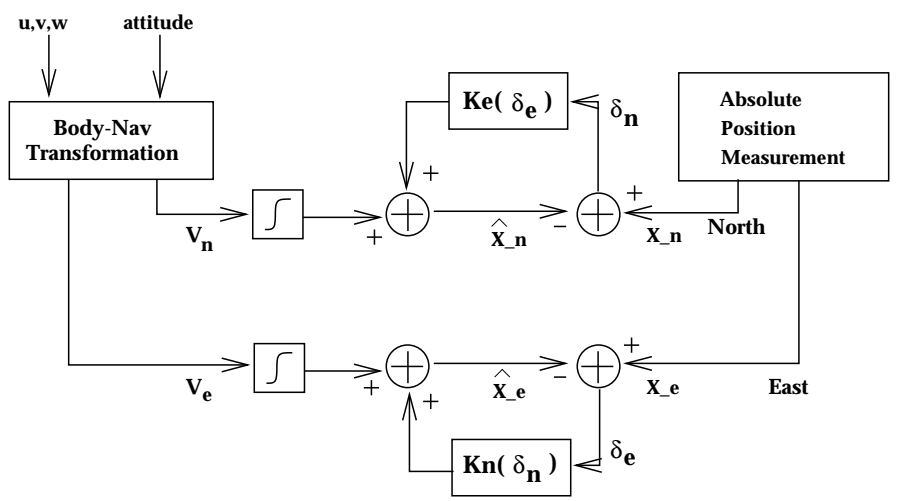

Figure 2: Closed-loop heuristic position estimator.

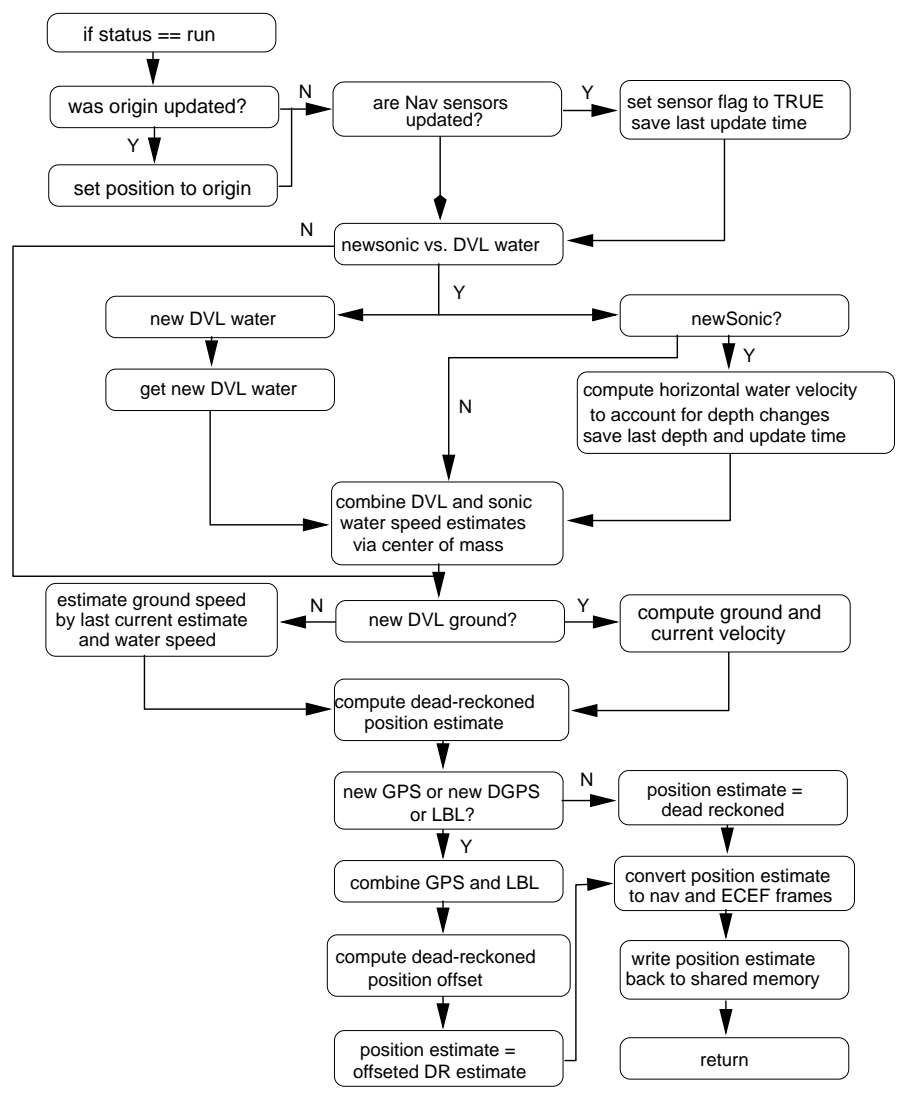

Figure 3: A flow chart of the position estimator algorithm.
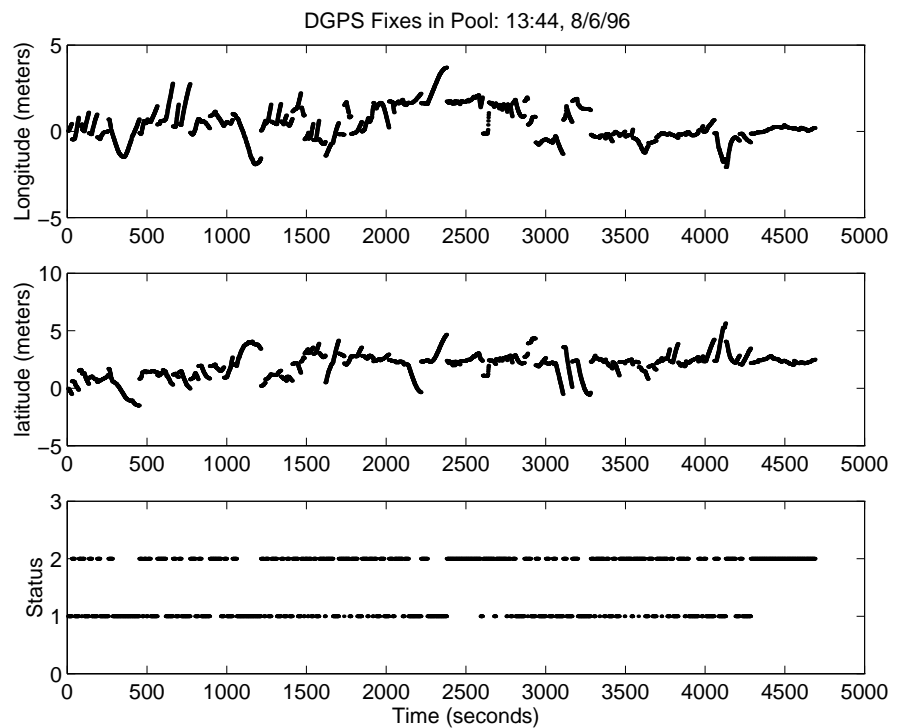

Figure 4: Motorola GPS time-history error evaluation. 


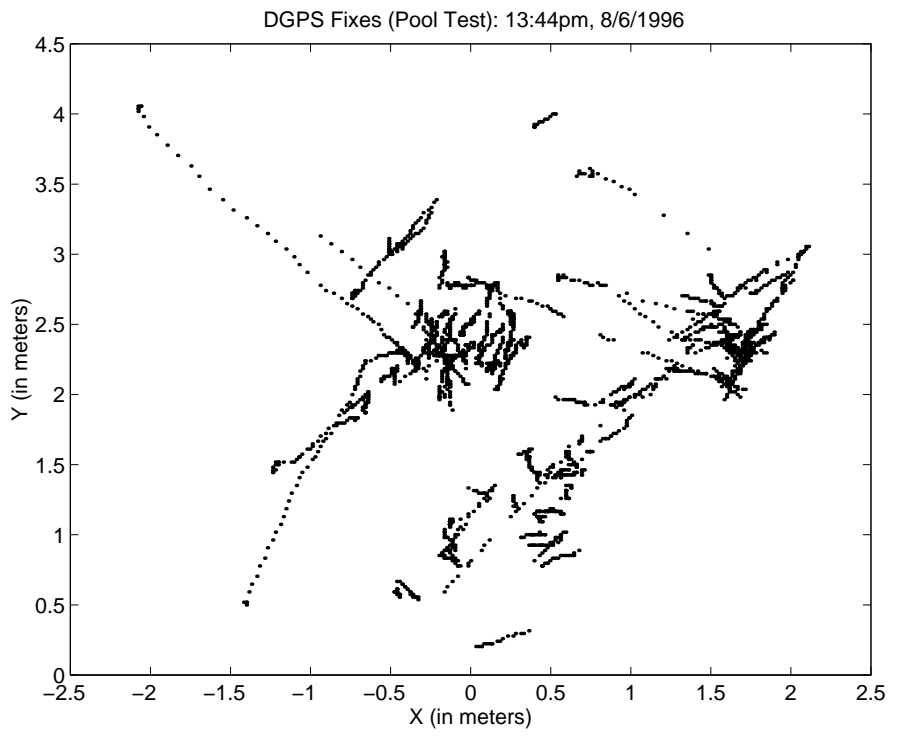

Figure 5: Motorola GPS X-Y error evaluation.

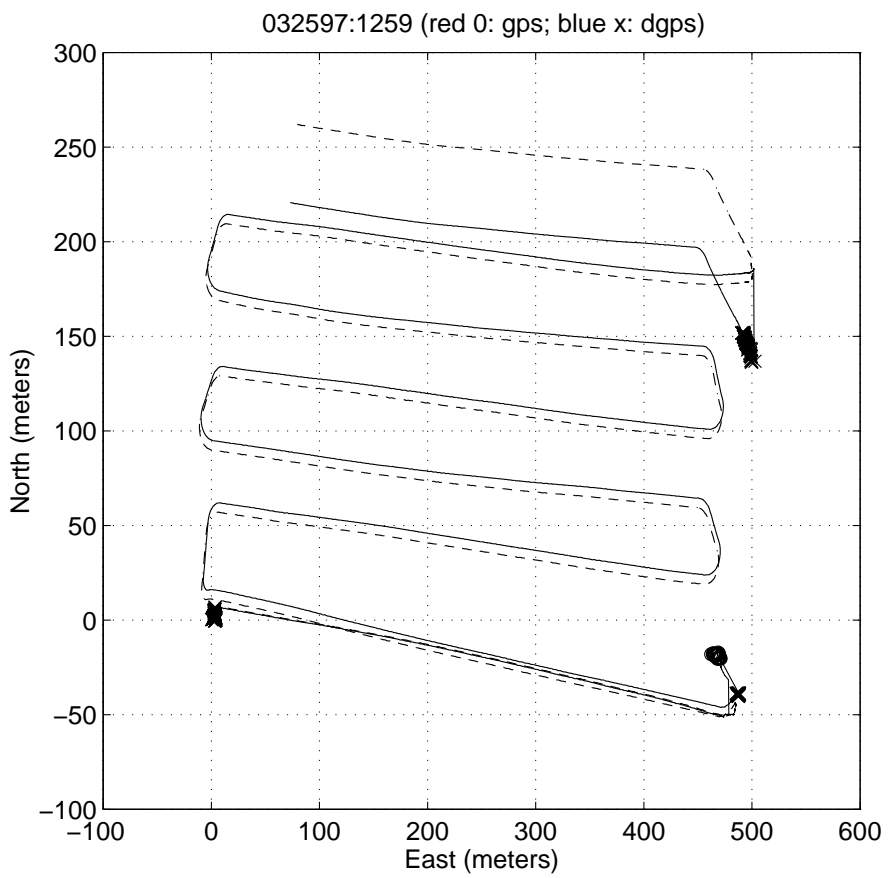

Figure 6: Heuristic position estimator and GPS outputs. Solid line: heuristic position estimator output, and dash line: dead-reckoned output. ' $\mathrm{X}$ ' and 'O' represent differential and regular GPS fix respectively.

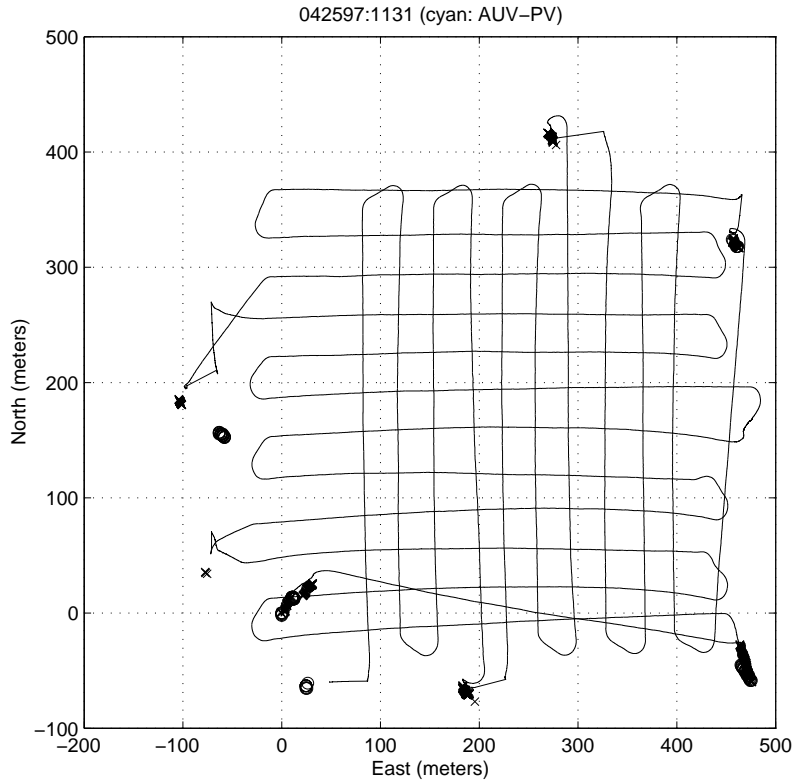

Figure 7: Heuristic position estimator and GPS outputs. Solid line: heuristic position estimator output, and dash line: dead-reckoned output. ' $\mathrm{X}$ ' and 'O' represent differential and regular GPS fix respectively. 